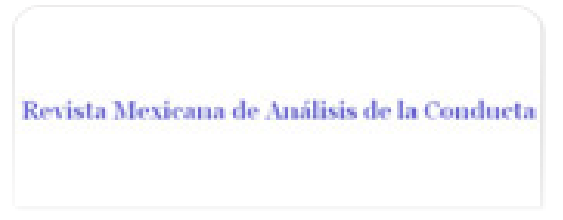

Revista Mexicana de Análisis de la Conducta ISSN: 0185-4534

editora@rmac-mx.org

Sociedad Mexicana de Análisis de la Conducta México

Espinosa R., Julio; Nieto, Javier; Campuzano, Nissyen; Pérez, Marcel

Discriminación fina de respuestas en palomas

Revista Mexicana de Análisis de la Conducta, vol. 31, núm. 2, diciembre, 2005, pp. 189-213

Sociedad Mexicana de Análisis de la Conducta

Guadalajara, México

Disponible en: http://www.redalyc.org/articulo.oa?id=59331201

Cómo citar el artículo

- Número completo

- Más información del artículo

- Página de la revista en redalyc.org

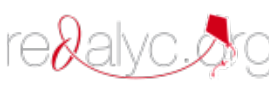

Sistema de Información Científica

Red de Revistas Científicas de América Latina, el Caribe, España y Portugal Proyecto académico sin fines de lucro, desarrollado bajo la iniciativa de acceso abierto 


\title{
DISCRIMINACIÓN FINA DE RESPUESTAS EN PALOMAS
}

\author{
FINE RESPONSE DISCRIMINATION IN PIGEONS \\ JULIO ESPINOSA R., JAVIER NIETO, \\ NISSYEN CAMPUZANO Y MARCEL PÉREZ ${ }^{1}$ \\ FACULTAD DE PSICOLOGÍA \\ UNIVERSIDAD NACIONAL AUTÓNOMA DE MÉXICO
}

\begin{abstract}
RESUMEN
Se presentan los resultados de dos experimentos sobre discriminación fina de respuestas en palomas. En ambos experimentos se entrenaron a palomas a picar una tecla bajo un programa de Número Fijo Consecutivo (NFC); en el que la emisión de un número mínimo de respuestas antes de visitar el comedero fue reforzada. El Experimento 1 utilizó dos requisitos de respuesta: NFC 4 y NFC 8, y evaluó los efectos del orden de su presentación. Los resultados revelaron que las palomas que iniciaron con un NFC 4 para después cambiar al NFC 8, mostraron una mejor discriminación de la cantidad de respuestas necesaria para obtener el reforzador que las palomas que iniciaron con la secuencia inversa. El propósito del Experimento 2 fue probar, mediante un programa NFC con límites inferior y superior del requisito de respuesta, un procedimiento de aproximaciones sucesivas al requisito exacto del programa, seguido de un cambio en los valores de esa condición: para algunas palomas el cambio consistió en un incremento y para otras una reducción en el
\end{abstract}

1. Los experimentos fueron parte del trabajo de investigación del primer autor para obtener el grado de Doctor en Psicología de la UNAM, con la beca CONACYT 158728. Asimismo, fueron parte del proyecto de investigación CONACYT 40849-H dirigido por el Dr. Javier Nieto (janigu@servidor.unam.mx). Los autores agradecen a Diana Herrera, Gustavo Bachá, Erika Gutiérrez y Livia Sánchez por sus contribuciones durante la presente investigación. Toda correspondencia relacionada con el artículo deberá enviarse al primer autor: Facultad de Psicología, UNAM, Edificio C cubículo 6, Av. Universidad 3004. Copilco Universidad, 04510 México, D.F. (correo electrónico: julioe@servidor.unam.mx). 
número exacto de respuestas. La discriminación fue mejor en los sujetos que cambiaron a un número mayor de respuestas en la segunda condición. Estos datos apoyan la tesis de que las palomas discriminan de manera diferencial entre cantidades adyacentes de elementos.

Palabras clave: programa NFC, conteo, discriminación numérica, respuesta a la tecla, palomas.

\begin{abstract}
The results of two experiments on fine response discrimination in pigeons are presented. In both experiments pigeons were exposed to a Fixed Consecutive Number (FCN) schedule which required to peck a key for a fixed number of times and then visit the food hooper. Experiment 1 used two schedules (FCN 4 and FCN 8) and assessed the effects of their presentation order. The results showed that pigeons that were first trained on a FCN 4 schedule and then switched to a FCN 8 showed a better performance than pigeons exposed to the reversed sequence of conditions. The purpose of Experiment 2 was to test, using a FCN schedule with fixed upper and lower response requirements, a shaping procedure which consisted in gradually reducing the upper and the lower limits to the exact number of responses; pigeons were changed to a higher or lower FCN schedule. Results showed that response discrimination was better in the subjects that werw changed to a high FCN schedule. These data support the thesis that pigeons discriminate in a differential way among adjacent quantities of elements.

Key words: FCN schedule, counting, numerical discrimination, responses on key, pigeons.
\end{abstract}

Existe evidencia de la capacidad de los animales para discriminar aspectos cuantitativos tanto del ambiente como de su propia conducta (Davis y Pérusse, 1988; Gallistel y Gelman, 1992). Se ha observado, por ejemplo, que las ratas son capaces de producir una cantidad mínima de respuestas para obtener un reforzador (Mechner, 1958a), que discriminan conjuntos de estímulos auditivos y visuales, numerosidades como le han denominado Meck y Church (1983) y que los monos tienen la habilidad de desarrollar relaciones entre símbolos arbitrarios y numerosidades (Matzusawa, 1985).

La posibilidad de que los animales utilicen tales competencias en su medio natural se considera una situación especulativa. Sin embargo, no es difícil encontrar circunstancias en las que esa discriminación proporcione a los animales información ecológica relevante. En situaciones de búsqueda de alimento, la sensibilidad para realizar discriminaciones de ese tipo podría 
facilitar la detección de cambios en la tasa de pago de los recursos disponibles en las distintas fuentes de alimentación (Krebs, 1978). La sensibilidad para detectar cambios en la densidad también es útil si un animal se ve en la necesidad de analizar el tamaño de un conjunto de predadores, o bien, si requiere examinar el ordenamiento de los machos dominantes en su propio grupo (Hauser, 2000).

En ambientes controlados la discriminación numérica en animales se ha analizado mediante dos estrategias. La primera estudia la discriminación entre conjuntos de estímulos presentados secuencial o simultáneamente; en la segunda se investiga la relación entre las propiedades discriminativas de los programas de reforzamiento y la propia conducta (para una revisión completa de ambas estrategias, ver Davis y Memmott, 1982; Davis y Pérusse, 1988). Los procedimientos experimentales empleados en el último caso, son derivados de programas de reforzamiento Razón Fija (RF) cuyo criterio para la entrega del reforzador es la emisión de un número determinado de respuestas.

Un arreglo procedente de los programas de razón es el denominado Número Fijo Consecutivo, o NFC (Mechner, 1958a). En este procedimiento el sujeto debe emitir un número mínimo de respuestas sucesivas a una palanca (palanca de carrera) y después emitir una sola respuesta a otra palanca (palanca de reforzamiento) para obtener el reforzador. Si el sujeto responde a la palanca de reforzamiento antes de completar un número mínimo de respuestas en la palanca de carrera, el reforzador es retenido y el contador asociado a la palanca de carrera reinicia en cero. En general, los datos obtenidos con esta preparación muestran que la instalación de un operando adicional para finalizar el ensayo produce cierta variabilidad en la longitud de carrera de respuesta (Hurwitz, 1962; Platt y Johnson, 1971).

En la investigación pionera sobre discriminación numérica de la propia conducta, Mechner (1958a) manipuló la proporción de ensayos de programas RF y NFC durante la sesión, y los valores de éstos $(4,8,12,16)$ fueron presentados en ese orden y en condiciones independientes. Los resultados de las ratas mostraron distribuciones normales y simétricas de las longitudes de la carrera de respuesta, con modos un poco más grandes que el valor del programa en cada condición. Un hallazgo adicional fue que los incrementos en $\mathrm{N}$ produjeron una mayor variabilidad en la longitud de la carrera antes de ingresar al comedero. Mechner interpretó los resultados considerando que en los programas NFC se refuerza el cambio a la palanca de reforzamiento bajo ciertos estímulos discriminativos producidos por la respuesta. Sin embargo, es difícil comprender esa afirmación, ya que en los programas NFC no existe cambio de estimulación alguno en la cámara experimental, sino hasta que el sujeto finaliza el ensayo; a no ser que Mechner se refiriera a estímulos generados por la propia conducta.

Las distribuciones de las longitudes de carrera de respuesta observadas 
en el experimento de Mechner (1958a), han sido replicadas consistentemente en estudios semejantes a éste. Mechner y Guevrekian (1962) investigaron los efectos de la privación de líquido en ratas mediante el empleo de dos programas de reforzamiento, uno de los cuales fue NFC, y el otro un procedimiento experimental, utilizado en el estudio de la conducta de estimación temporal, conocido como de intervalo fijo mínimo (IFM). En el caso de Snodgrass, Hardin y McMillan, (1997), se analizó en ratas el efecto de drogas como diazepam, morfina y pentobarbital, con la tarea de completar un mínimo de 16 respuestas en un programa NFC. El resultado fue prácticamente el mismo: una producción de carreras de respuesta con una función semejante a la de una distribución normal y con una mayor frecuencia de éstas sobre o alrededor del valor de $\mathrm{N}$.

Platt y Johnson (1971) con el propósito de observar el efecto de terminar el ensayo antes de cumplir con el requisito de respuesta sobre la distribución de las longitudes de carrera, expusieron a un grupo de ratas a un programa NFC y a otro a un programa conocido como programa de Número Fijo, o NF. En este procedimiento, a diferencia de lo que ocurre en los programas NFC, finalizar los ensayos antes de emitir la cantidad mínima de respuestas no regresa el contador de respuestas a cero. Los resultados en la condición NFC mostraron que la producción de distribuciones fue simétrica en la longitud de carrera, con modas ligeramente por arriba del valor del programa. Las distribuciones en la condición NF fueron también simétricas, pero con un sesgo negativo creciente para los valores mayores de N. Es decir, bajo la condición NF hubo una tendencia a subestimar el requisito del programa cuando los "errores" en el número de respuestas necesarias para obtener el reforzador no fueron castigadas con el regreso a cero del contador de respuestas.

Los resultados conjuntos de Platt y Johnson (1971) y Hurwitz (1962), señalan a la "contingencia del error" como un factor determinante en la ejecución en programas NFC. En otras palabras, y de acuerdo a la conclusión más general del estudio de Platt y Johnson (1971), la discriminación numérica de la propia conducta es más precisa en la medida en que la contingencia para obtener el reforzador lo demanda.

Otras investigaciones más recientes, no relacionadas directamente con el presente estudio, (Beninger, Kendall y Vanderwolf,1974; Shimp,1982, 1983; Lionello-DeNolf y Urcuioli, 2003) revelan la presencia del responder diferencial sin ayuda de estímulos exteroceptivos.

En resumen, los resultados mencionados son ejemplos de la discriminación basada en las características numéricas del comportamiento, en los que se enfatiza la importancia de los procedimientos experimentales utilizados para desarrollar tal discriminación.

El propósito general de la investigación que se reporta fue probar si mediante el entrenamiento adecuado las palomas son capaces de desarrollar 
una discriminación numérica fina o exacta de su propia conducta, en términos del número de respuestas necesarias para obtener el reforzador.

\section{EXPERIMENTO 1}

Una característica de los programas NFC es que solamente establecen el requisito mínimo de respuesta antes de cambiar y responder a la palanca alternativa. Si el sujeto responde a la palanca de reforzamiento antes de completar el número mínimo de respuestas en la palanca de carrera, el reforzador se omite y el contador asociado a la palanca de respuesta regresa a cero. Por consiguiente, es posible que este procedimiento favorezca la discriminación del mínimo de respuestas y no una discriminación numérica precisa. En consecuencia, el propósito de este experimento fue el análisis de la distribución de la longitud de la carrera de respuestas ante una manipulación reversible en el requisito de respuesta de un programa NFC. La razón para incluir una condición en la que el número mínimo de respuestas disminuya durante la segunda fase en relación al primer valor, se justifica debido a que una condición semejante es utilizada en algunos sujetos de las fases de prueba del Experimento 2, donde se requiere que los sujetos emitan un número exacto de respuestas menor al número establecido en la fase previa.

El diseño permitió observar el desarrollo del ajuste conductual a los valores del programa. Es decir, un par de palomas fueron expuestas primero a un programa NFC 4 y en una siguiente fase a un NFC 8, mientras que otro par de palomas fue expuesto a la secuencia inversa. No obstante la ausencia de estudios con programas NFC con diseños reversibles, se esperaría que las palomas expuestas a la transición del NFC 8 al NFC 4 mantuvieran su ejecución de la primera fase, ya que presentando un mínimo de 8 respuestas durante el resto de las fases experimentales asegurarían un desempeño exitoso. En el caso de la transición de NFC 4 a NFC 8, la regla es simple: incrementar el número de respuestas.

Adicionalmente, en este experimento se utilizó una modificación del procedimiento de Mechner (1958a). En éste, la rata debía emitir un número mínimo de respuestas sucesivas a una palanca (palanca de carrera) y después emitir una sola respuesta en otra palanca (palanca de reforzamiento) para obtener el reforzador. En el presente experimento se instaló una fotocelda en la entrada del comedero, el haz de luz era interrumpido cuando la paloma introducía la cabeza al comedero. La inserción de la cabeza sustituyó a la segunda respuesta en el procedimiento de Mechner (1958a). 


\section{MÉTODO}

Sujetos: Cuatro palomas de aproximadamente tres años de edad al inicio del experimento, ingenuas experimentalmente, provistas por el bioterio de la Facultad de Psicología de la UNAM. Las palomas fueron privadas de alimento y mantenidas al $80 \%$ de su peso ad libitum, con acceso al agua durante todo el experimento.

Aparatos: Se usaron dos cámaras experimentales para palomas fabricadas por Med Associates ${ }^{\circledR}$ Mod. ENV-007. Cada cámara estuvo equipada con tres teclas centradas sobre un panel frontal de aluminio a $22 \mathrm{~cm}$ por encima del piso, cada una de $2 \mathrm{~cm}$ de diámetro, y con una separación de $8 \mathrm{~cm}$ de centro a centro. La tecla central, única en operación, requirió de una fuerza para accionarla de $15 \mathrm{~g}$ y se iluminaba con una luz blanca de $7.5 \mathrm{w}$. Directamente debajo de la tecla central, y a $4 \mathrm{~cm}$ del piso, se situó un dispensador movible de alimento de $6 \times 7 \mathrm{~cm}$. De manera transversal a éste; se hizo pasar un rayo fotoeléctrico a través de un orificio de $1 \mathrm{~cm}$ detrás de la pared para registrar el ingreso de las palomas al comedero. Sobre la pared trasera de la caja, un foco de $7.5 \mathrm{w}$ produjo la iluminación general. Cada una de las cámaras experimentales fueron colocadas dentro una caja amortiguadora del sonido externo con un ventilador que produjo ruido blanco. Para el control de los eventos y registro de las respuestas de los sujetos, se utilizó una computadora Acer 486, conectada a una interfase Med Associates ${ }^{\circledR}$ Mod. SG6080D. Las respuestas y sus tiempos de ocurrencia se registraron con una aproximación a la décima de segundo.

Procedimiento: Los sujetos fueron asignados al azar a dos condiciones con dos animales cada una, y sometidos a dos valores de un programa NFC. Se utilizó un diseño experimental reversible, el orden de las condiciones experimentales, así como el número de sesiones por fase para cada sujeto, se muestran en la Tabla 1. Las palomas expuestas a la condición Ascendente (C) iniciaron con un valor del programa NFC 4, y, en la siguiente fase, fueron expuestas a un NFC 8. Los animales en la condición Descendente (D) iniciaron con NFC 8 y fueron cambiados después a NFC 4.

Cada sesión terminaba al cumplirse 100 ensayos, reforzados o no. Cada ensayo inició con el encendido simultáneo de las luces general y de la tecla central, y finalizó cuando la paloma visitaba el comedero e interrumpía el haz de luz de la fotocelda. Si el ensayo era reforzado, las luces general y de la tecla se apagaban, encendiéndose la luz ubicada en el área del comedero, permitiendo así el acceso al grano durante tres segundos. Cuando el ensayo no fue reforzado se programó un apagón general de 10 segundos de duración. Las condiciones de este intervalo entre ensayos fueron las mismas para los ensayos reforzados. Cada paloma fue expuesta a esta condición por lo menos 15 sesiones. El criterio de cambio de fase dependió de que la fre- 
cuencia de respuestas se situara alrededor del valor requerido durante cinco sesiones consecutivas.

Tabla 1. Muestra el orden de las condiciones experimentales para cada grupo. En la fila superior se muestra el sujeto y el grupo al que pertenece $(C, D)$; para cada fase se muestra el valor del programa NFC y entre paréntesis el número de sesiones por condición durante el Experimento 1.

\begin{tabular}{|c|c|c|c|c|}
\hline Fase & C J 53 & C J 22 & D J 17 & D J 94 \\
\hline 1 & $(30) 4$ & $(38) 4$ & $(44) 8$ & $(60) 8$ \\
\hline 2 & $(29) 8$ & $(36) 8$ & $(25) 4$ & $(42) 4$ \\
\hline 3 & $(21) 4$ & $(32) 4$ & $(22) 8$ & $(21) 8$ \\
\hline 4 & $(18) 8$ & $(18) 8$ & $(15) 4$ & $(17) 4$ \\
\hline
\end{tabular}

\section{RESULTADOS}

Para las palomas de la condición C la Figura 1 muestra la probabilidad de que los sujetos cambien sus respuestas en la tecla para ingresar al comedero después de diferentes longitudes de carrera. En la columna de la izquierda se presentan las gráficas correspondientes a las probabilidades de ocurrencia durante los primeros cinco días y en la columna derecha están las gráficas relacionadas con las probabilidades de ocurrencia de los últimos cinco días de cada condición. Se observa una ejecución semejante en ambos sujetos, caracterizada por un pico alrededor del valor del programa. El valor de las probabilidades en la condición de reversión, es mayor si se compara con los resultados obtenidos en la primera ocasión en que los sujetos fueron expuestos a un valor determinado del programa NFC. No obstante lo anterior, las gráficas de los primeros cinco días muestran un rápido ajuste en las transiciones de un valor NFC al nuevo valor de éste. En la Figura 2, de la misma manera que en la Figura 1, se muestran las probabilidades de ocurrencia de las diferentes longitudes de carrera de los sujetos en la condición D. Para estas palomas el valor inicial del programa NFC fue 8 . En ambos sujetos la variabilidad en la probabilidad para todas las condiciones fue mayor a la obtenida en la condición $C$, por esta razón los valores máximos en la probabilidad de ocurrencia de la longitud de la carrera se ubican por arriba del valor mínimo de respuestas, o valor del programa.

La forma en que se distribuye la probabilidad de ocurrencia de las longitudes de la carrera antes de interrumpir la fotocelda es un dato primario, sin embargo, el uso de la probabilidad condicional provee de un índice complementario de discriminación. En este caso se considera a la probabilidad con- 


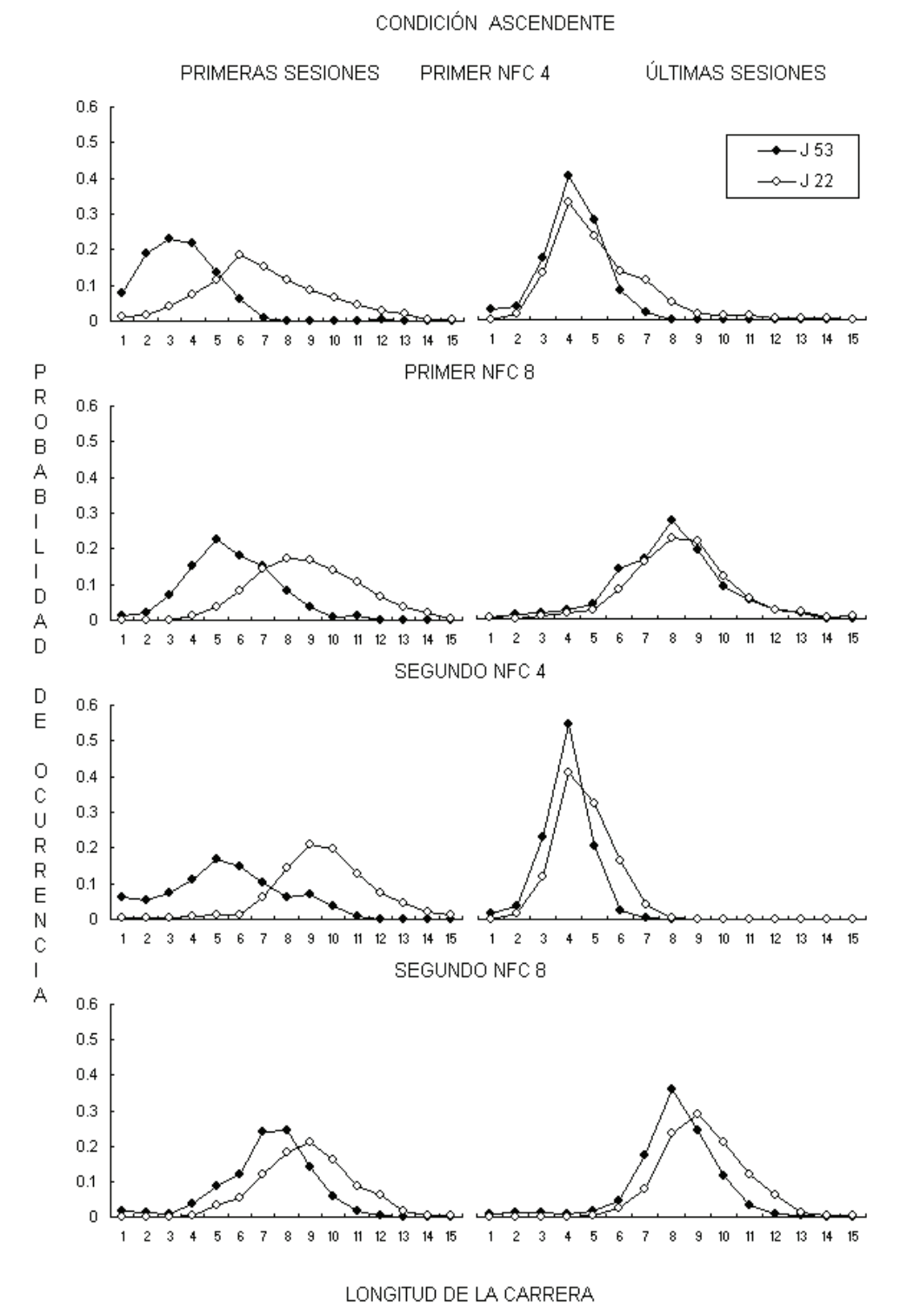

Figura 1. Muestra la probabilidad de cambiar de la tecla al comedero después de carreras de distintas longitudes para el grupo Ascendente en los primeros cinco días y los últimos de cada fase del Experimento 1. 
CONDICIÓN DESCENDENTE

PRIMERAS SESIONES PRIMER FCN 8 ULLTIMAS SESIONES
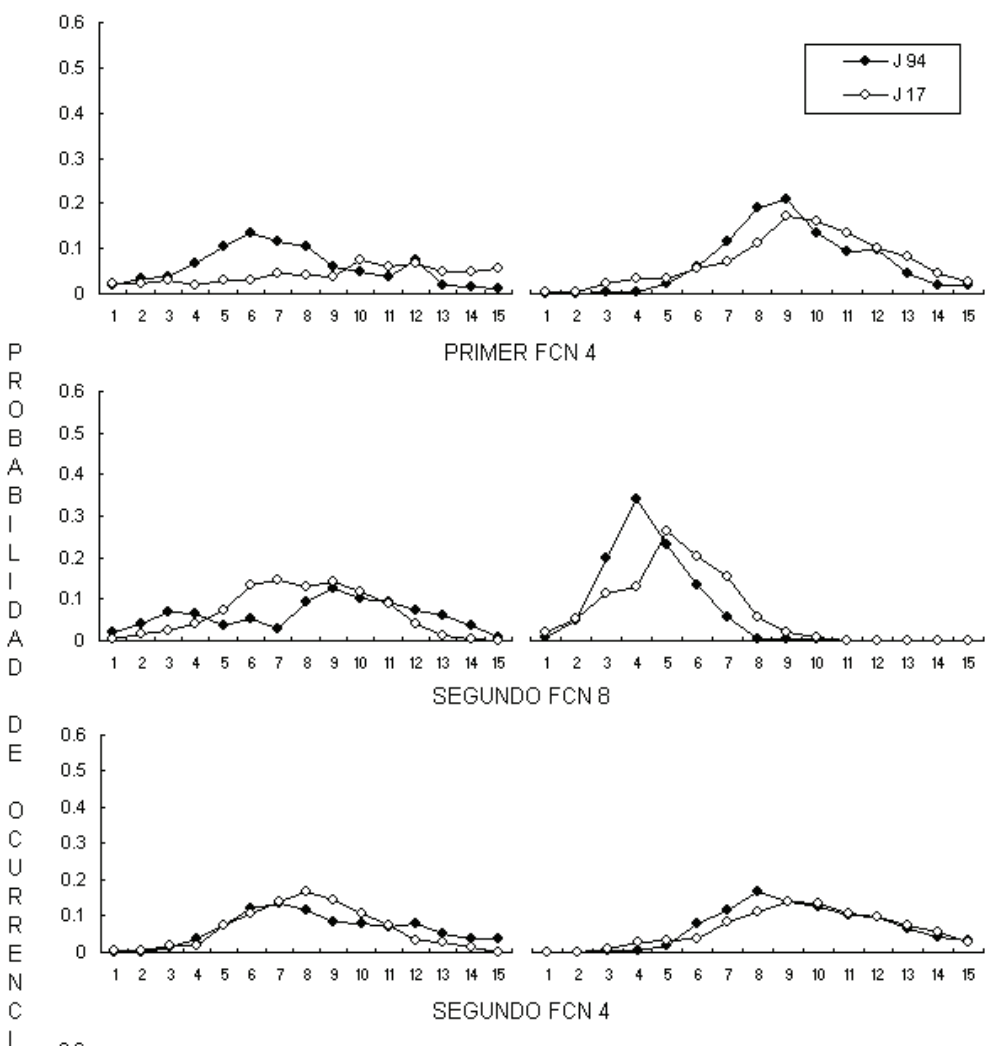

A

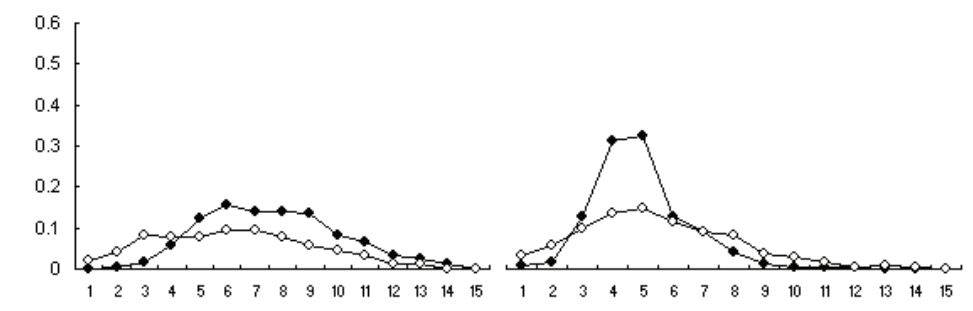

LONGITUD DE LA CARRERA.

Figura 2. Muestra la probabilidad de cambiar de la tecla al comedero después de carreras de distintas longitudes para el grupo Descendente en los primeros cinco días y los últimos días de cada fase del Experimento 1. 
dicional como la probabilidad de que habiendo realizado ya $\mathrm{N}$ respuestas, el sujeto emita una más. De tal manera que como un índice de buena discriminación, conforme los sujetos se aproximen al valor del programa se esperaría que la probabilidad de emitir una respuesta más decrementara.

La Figura 3 muestra la probabilidad condicional para las primeras y últimas cinco sesiones de cada condición del grupo C. En general se observa en ambos sujetos una reducción drástica en la probabilidad de emitir una respuesta más, conforme la cantidad emitida de respuestas por los animales se aproxima al número mínimo establecido en el programa NFC. Por ejemplo, en los últimos días de la condición de reversión en NFC 4, la probabilidad de que la paloma $\mathrm{J} 53$ responda una vez más antes de ir al comedero habiendo emitido 3 respuestas fue $p=.74$, para 4 respuestas $p=.36$, y para 5 respuestas $p=.20$. En otras palabras, habiendo emitido 4 respuestas la probabilidad de emitir una más antes de ir al comedero fue prácticamente nula. Las funciones de la paloma $\mathrm{J} 22$ son prácticamente simétricas a las funciones del sujeto J 53. Los datos para la condición de reversión en NFC 8, muestran un índice de discriminación semejante.

Resumiendo, las pendientes que describen las funciones de la probabilidad condicional son prácticamente simétricas en los sujetos de la condición C; este efecto es más notable en las funciones correspondientes a los últimos días de cada condición.

En la Figura 4 se presenta la probabilidad condicional de los sujetos J 94 y J 17 en la condición D. Al comparar las probabilidades de los primeros y los últimos días en cada valor del programa NFC, se observa que la discriminación es menos precisa que en la condición $C$, aunque en las últimas sesiones de cada condición la discriminación mejora sensiblemente.

En las Figuras 5 y 6 se presentan los datos de los últimos cinco días de cada condición experimental. En la Figura 5 se muestran, por sujeto, los promedios de la carrera de respuesta para los valores del programa NFC y el coeficiente de variación correspondientes a la condición C. Los promedios muestran un valor cercano al número mínimo de respuestas por reforzador en cada fase experimental. En el caso del coeficiente de variación, un valor constante de esta medida refleja la relación directa entre la magnitud del número mínimo a discriminar y la variabilidad de la respuesta. El resultado en este caso, es que los valores del coeficiente varían muy poco a través de las condiciones, lo que implica una mayor sensibilidad ante los cambios de los valores del programa NFC. En las gráficas de la Figura 6 se muestran los resultados correspondientes a los sujetos de la condición $\mathrm{D}$. Los datos de estos sujetos son muy semejantes a los mostrados por las palomas en la condición C. Quizá la única diferencia entre ambas condiciones, consiste en que los valores promedio de respuesta para los sujetos de esta condición se ubican un poco más lejos del valor mínimo de respuestas por reforzador. 


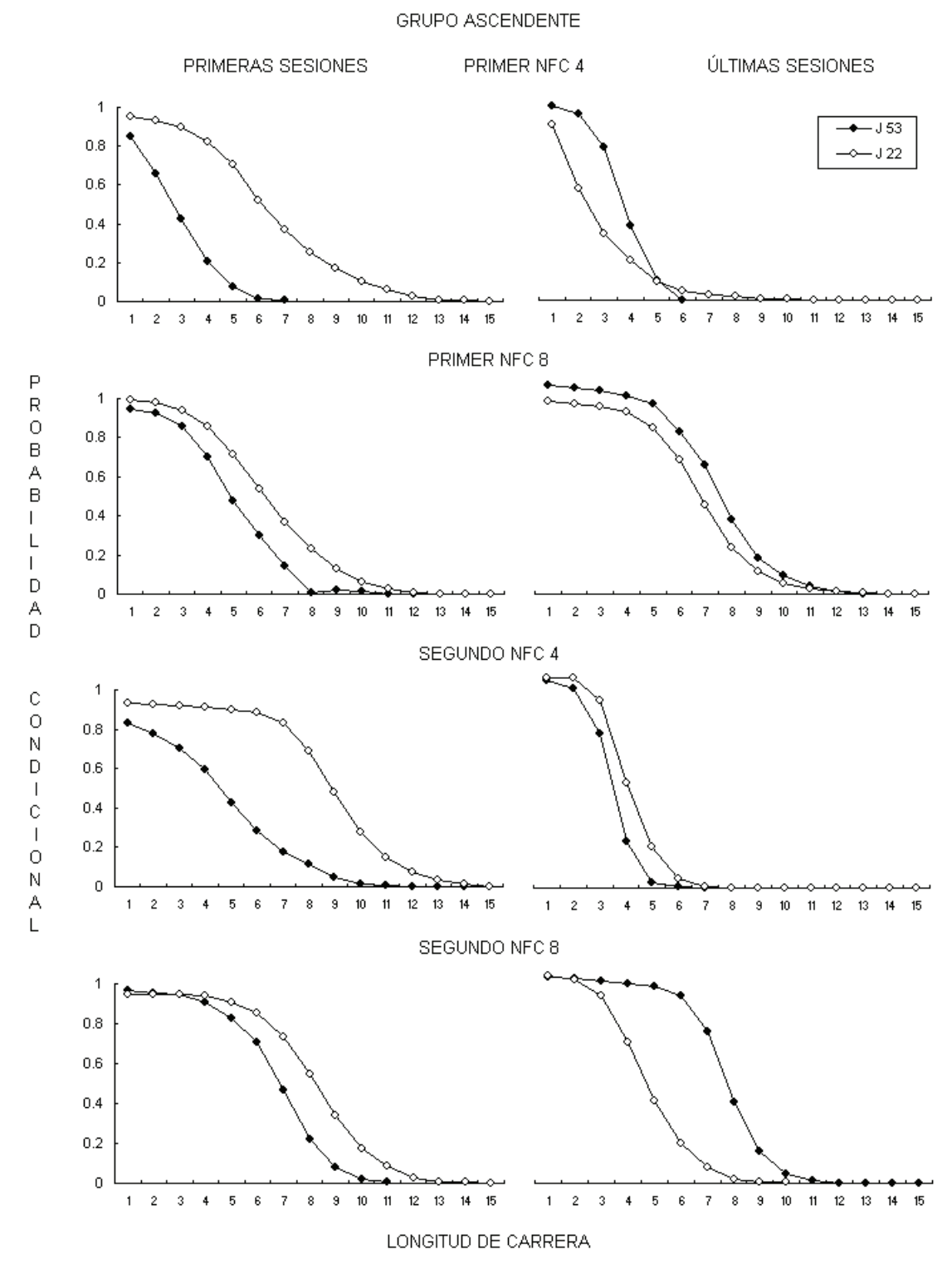

Figura 3. Se presenta la probabilidad condicional de que una paloma realice una o más respuestas habiendo emitido $N$ respuestas en la condición Ascendente del Experimento 1. 


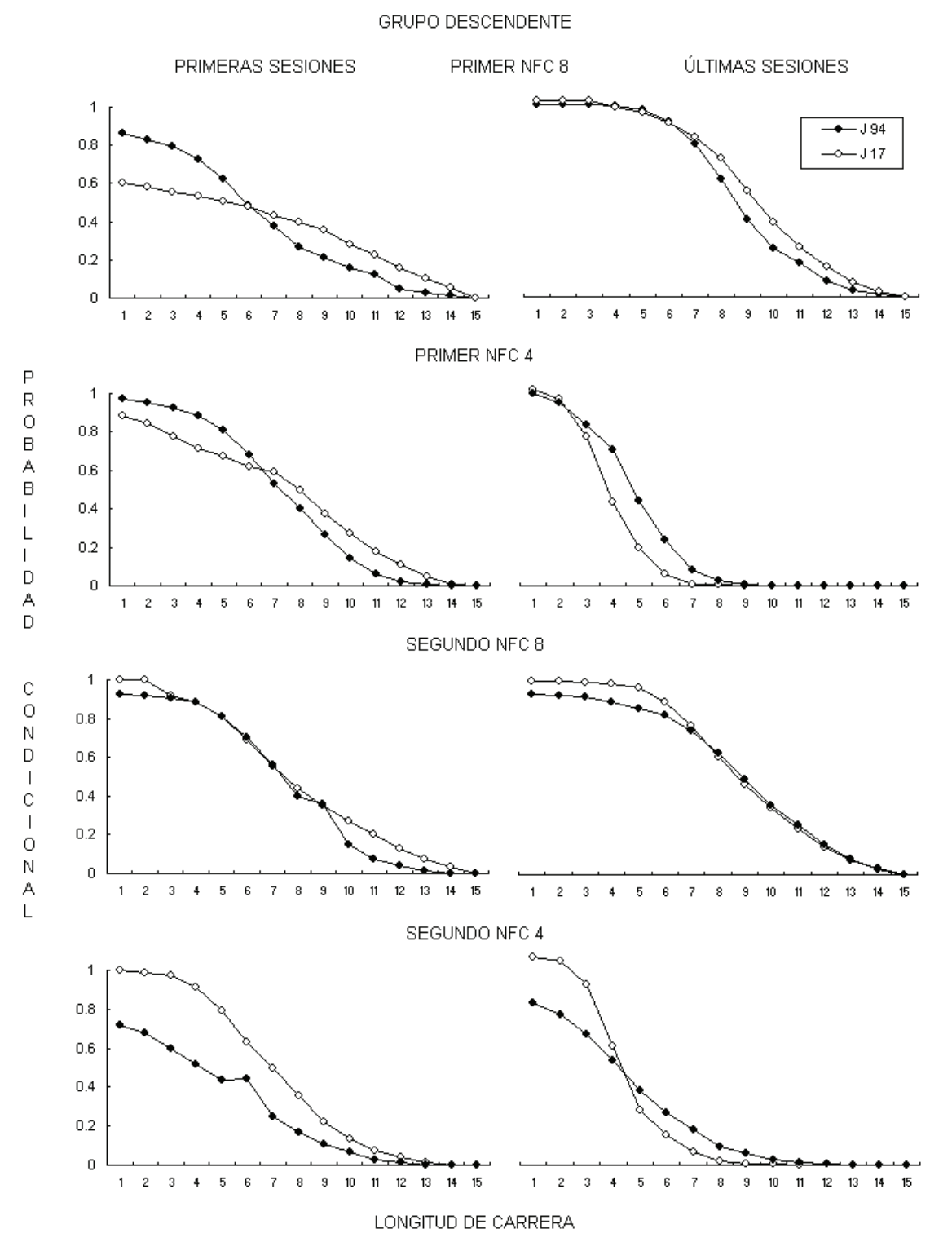

Figura 4. Se presenta la probabilidad condicional de que una paloma realice una o más respuestas habiendo emitido $N$ respuestas en la condición Descendente del Experimento 1. 

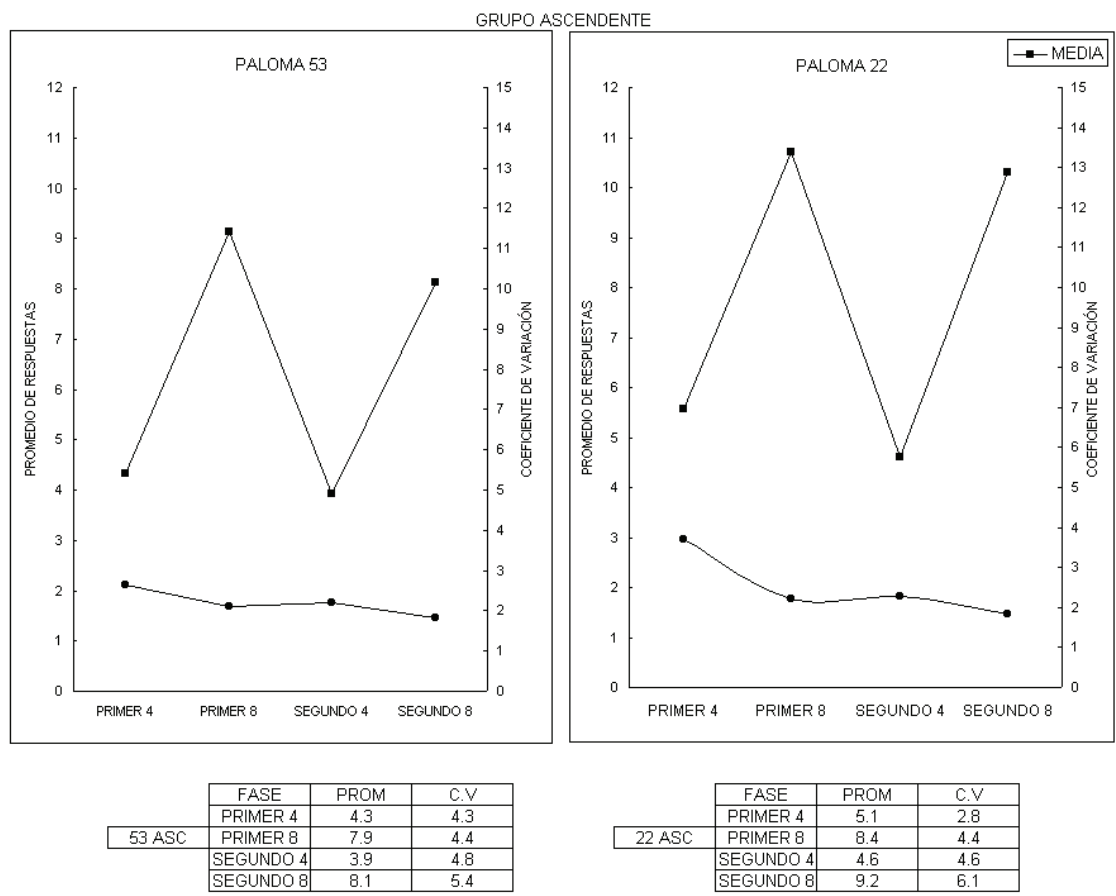

Figura 5. Muestra conjuntamente el promedio de respuestas y el coeficiente de variación de las carreras de respuesta para los sujetos de la condición Ascendente, correspondiente a los últimos cinco días de cada fase experimental, para las cuatro condiciones experimentales del Experimento 1.

\section{DISCUSIÓN}

La manipulación del orden de presentación de los valores del programa NFC, generó, en los sujetos de la condición C, que la distribución de las longitudes de las carreras de respuesta estuviera alrededor de los valores del programa actual. Este hallazgo, consistente con los obtenidos en investigaciones previas (Mechner, 1958a; Mechner y Guevrekian, 1962; Mechner y Latranyi (1963); Platt y Johnson, 1971), se observó con menor precisión en los sujetos de la condición $\mathrm{D}$. Al comparar los primeros cinco días de cada transición en la columna izquierda de las Figuras 1 y 2 , se observa que los sujetos de la condición $\mathrm{C}$, ajustaron más rápidamente la cantidad de respuestas emitidas al nuevo valor del programa. Si se compara con la ejecución observada en 

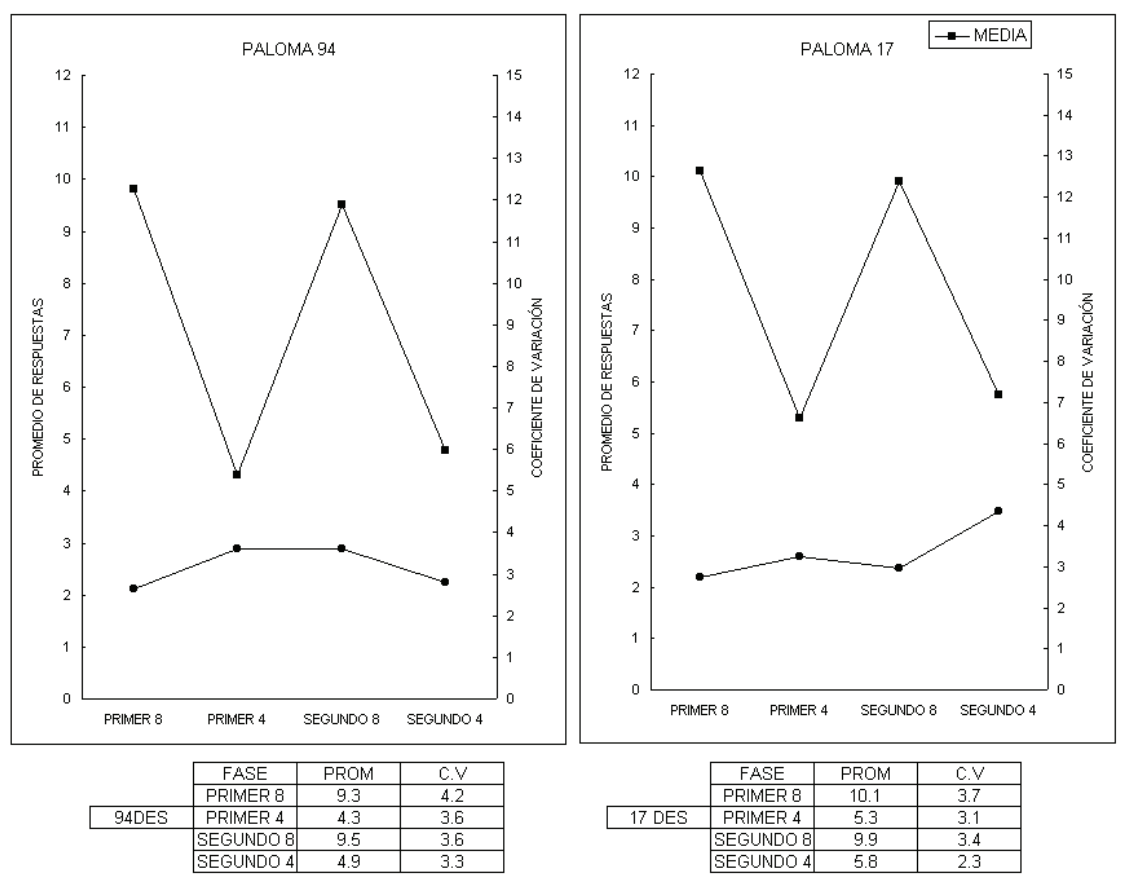

Figura 6. Muestra conjuntamente el promedio de respuestas y el coeficiente de variación de las carreras de respuesta para los sujetos de la condición Descendente, correspondiente a los últimos cinco días de cada fase experimental, para las cuatro condiciones experimentales del Experimento 1.

las palomas de la condición $\mathrm{D}$, las que, como se esperaba, muestran una probabilidad de ocurrencia de la carrera de respuesta muy semejante en todas las transiciones, (columna izquierda de la Figura 2) sin importar el valor actual del programa.

Los sujetos de la condición $D$ hubiesen podido enfrentar con éxito la tarea emitiendo siempre un mínimo de 8 respuestas desde la primera fase experimental, sin importar el valor del programa en las condiciones posteriores. Sin embargo, en la columna derecha de las Figuras 1 y 2 correspondientes a los últimos cinco días de cada condición, se observa que el valor más alto de la probabilidad de ocurrencia de una longitud de carrera en NFC 4, está muy próximo al requisito mínimo de respuestas.

Una pregunta que surge aquí es: ¿qué variable controló, en general, el ajuste asociado a cada una de las distintas condiciones experimentales? 
Para responder, debería partirse de la premisa de que la variable que controla la conducta en una tarea de discriminación es el reforzador. En la condición C, una vez que la ejecución se estabilizó en NFC 4 en la primera fase, la perseverancia en el responder ante un incremento en el mínimo de respuestas, tendría como resultado una mayor probabilidad de hacer contacto con el reforzador, incrementando también la probabilidad de ocurrencia de longitudes de carrera alrededor de NFC 8. Esta regla no aplicaría para los sujetos de la condición $\mathrm{D}$, para los que la única forma de discriminar el nuevo valor del programa era emitiendo una cantidad menor de respuestas antes de visitar el comedero; una vez que eso ocurre, la probabilidad de ocurrencia de longitudes de carrera cercanas a NFC 4 se ve fortalecida por el reforzador.

En resumen, los resultados del presente experimento muestran que el orden de presentación de las condiciones experimentales, en términos del valor del programa, afectó la discriminación numérica en palomas cuyo primer cambio requirió de una disminución en las respuestas y que esa dificultad fue más evidente en los primeros días de cada fase experimental. Por otra parte, los resultados permiten extender la generalidad de la discriminación numérica a preparaciones distintas a las empleadas hasta ahora. Este fenómeno ocurre si se utilizan dos palancas y ratas como sujetos (Mechner, 1958a; Hurwitz, 1962) o, si, como en el presente experimento, el procedimiento incluye la respuesta a la tecla y la interrupción de un rayo fotoeléctrico con cambios bidireccionales en los valores de los programas NFC, empleando palomas como sujetos.

\section{EXPERIMENTO 2}

A pesar de que en el Experimento 1 las palomas mostraron cierto ajuste respecto a la emisión del número mínimo de respuestas requeridas para obtener el reforzador, queda pendiente saber si la discriminación observada pudiera ser más precisa exigiendo la emisión de un número exacto de respuestas, sin embargo, utilizando un programa NFC convencional no es posible saberlo. Con este propósito se diseñó un segundo experimento para analizar si mediante el entrenamiento adecuado, las palomas son capaces de realizar una discriminación exacta del número de respuestas necesario para obtener el reforzador. Para ello, en una primera fase se establecieron límites superior e inferior a un procedimiento NFC, de tal manera que el número mínimo de respuestas a reforzar se ubicó dentro de un rango de éstas, para después probar la discriminación exacta. 


\section{MÉTODO}

Sujetos

Seis palomas de tres años de edad aproximadamente al inicio del estudio, con experiencia en programas de reforzamiento basados en la respuesta. Las condiciones de alojamiento y alimentación en el bioterio fueron las mismas que las del Experimento 1.

Aparatos

Los mismos utilizados en el Experimento 1.

Procedimiento

Debido a que los sujetos tenían experiencia en investigaciones sobre programas basados en la respuesta, se les expuso directamente a una condición en la que mediante un programa NFC, se programó que el reforzador fuera entregado sólo si el ingreso al comedero ocurría dentro de un rango inicial de respuestas entre 3 y 9 , es decir, que en la primera condición sólo fueron reforzados los ingresos al comedero precedidos de 3, 4, 5, 6, 7, 8 ó 9 respuestas en la tecla; en cualquier otra circunstancia de ingreso al comedero se programó un apagón de 10 segundos y el contador de respuestas para el siguiente ensayo regresó a cero. Es decir, a la contingencia del programa NFC se le agregó un límite superior y otro inferior en el número mínimo y máximo de respuestas antes de visitar el comedero.

El experimento constó de dos fases. En la fase de entrenamiento en rangos, las palomas fueron expuestas a un programa NFC que establecía límites inferiores y superiores de respuestas, NFC 3,9 por ejemplo, implica que todas las respuestas iguales o mayores a 3, pero no mayores a 9 eran reforzadas. El rango entre los límites fue reduciéndose gradualmente hasta alcanzar la unidad. Cada rango o condición estuvo vigente hasta que el número de reforzadores obtenidos fuera estable durante cinco sesiones consecutivas. El orden de las condiciones experimentales, así como el número de sesiones por fase para cada sujeto, se muestran en la Tabla 2. Cumplido ese criterio en la condición de 3 a 9 , los límites superior e inferior de respuestas fueron situados en 4 y 8 , y después en 5 y 7 .

Una vez concluido las condiciones de moldeamiento anterior, se estableció la fase de prueba; ésta consistió en exponer a las palomas a un valor exacto del programa NFC; en la que sólo fue reforzada la emisión de 6 respuestas antes de ir al comedero. Las palomas $\mathrm{J} 4$ y J 19 en esta condición experimental fueron retiradas de la investigación; la primera dejó de respon- 
Tabla 2. Muestra el orden de las condiciones experimentales y el número de sesiones para cada sujeto en el Experimento 2. En la fila superior se muestra el sujeto, el rango del programa NFC en la condición de entrenamiento y el valor del programa en la condición de exacto durante el Experimento 2. Para cada sujeto se muestra el número de sesiones que estuvo en cada rango y en las condiciones exactas.

\begin{tabular}{|c|c|c|c|c|c|}
\hline SUJETO & RANGO 3-9 & RANGO 4-8 & RANGO 5-7 & EXACTO 6 & EXACTO 8 \\
\hline J53 & 10 & 8 & 11 & 25 & 25 \\
\hline J24 & 7 & 5 & 46 & 25 & 25 \\
\hline J9 & 8 & 7 & 25 & 25 & 25 \\
\hline J12 & 17 & 22 & 62 & 25 & 25 \\
\hline J19 & 24 & 46 & 36 & & \\
\hline J4 & 9 & 31 & 24 & & \\
\hline
\end{tabular}

der y la segunda ingresó consistentemente al comedero sin emitir respuesta, por lo que los datos de estos sujetos se presentarán sólo hasta la condición de rangos. Toda vez que la ejecución se hubiese estabilizado en el valor de 6 exacto, los cuatro sujetos restantes fueron asignados al azar a dos condiciones de dos sujetos cada una. Para una de las condiciones el requisito de respuesta fue de 4 respuestas y en la otra condición fue de 8 . En las fases de entrenamiento y experimentales, el reforzador consistió de 3 segundos de acceso al grano, el intervalo entre ensayos, reforzados o no reforzados, fue de 10 segundos, y la sesión finalizó con el ensayo 100.

\section{RESULTADOS}

El análisis de los datos del Experimento 2 corresponde a los últimos cinco días de cada condición experimental. La Figura 7 muestra para todos los sujetos, los porcentajes correspondientes a las longitudes de carrera en el límite inferior de las fases de entrenamiento en rangos, así como los porcentajes de las condiciones del número exacto de respuestas de los sujetos que trabajaron en ellas. La razón de presentar los datos del límite inferior, es que la mayor probabilidad de ocurrencia, en prácticamente todas las condiciones, se ubicó en ese valor del rango. En la Figura 7 se observa que la distribución de los porcentajes de respuesta no sufrió variaciones importantes, no obstante las modificaciones en los valores de los rangos durante las distintas condiciones. En el caso de los cuatro sujetos que trabajaron en la condición de exactos, los porcentajes para el valor 6, fueron prácticamente los mismos sin importar que la siguiente condición de exacto fuera incrementar a 8 o decrementar a 4 el valor del programa. 


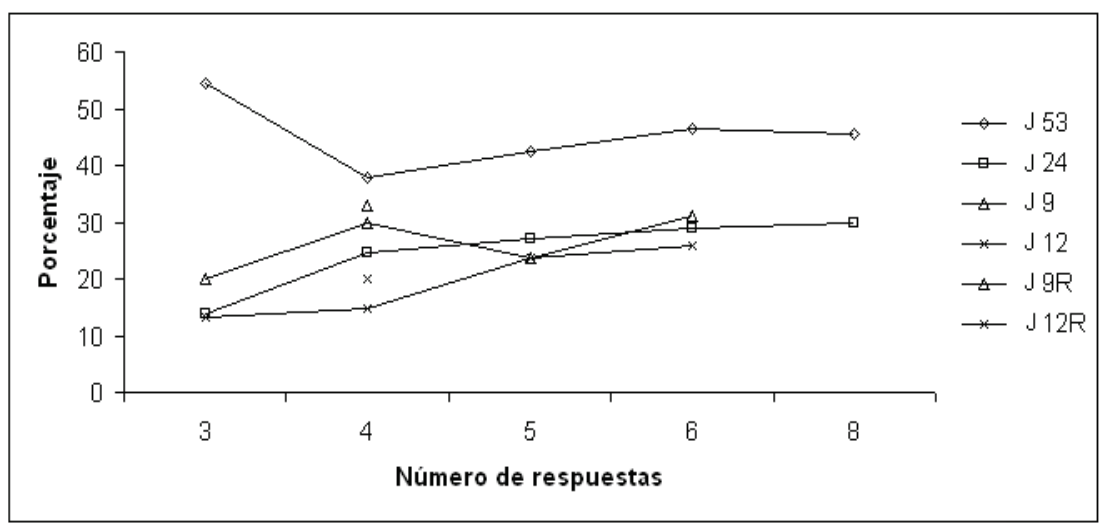

Figura 7. Muestra, para todos los sujetos, la distribución de los porcentajes de respuestas correspondiente al límite inferior de las condiciones de rangos, así como las condiciones de número exacto de respuestas para los sujetos que trabajaron en ellas. Los datos corresponden a los últimos cinco días de cada condición experimental en el Experimento 2.

La Figura 8 muestra la distribución del porcentaje de respuestas de los distintos valores de las longitudes de carrera. En la columna de la izquierda aparecen las gráficas de la condición de 6 a 8 exacto. En ambos sujetos, el mayor porcentaje se ubica sobre el valor del programa, tanto en la condición de 6 , como la de 8 respuestas. En la condición de 6 a 4 respuestas, el nivel de discriminación es semejante entre los sujetos, pero en la condición de 4 exacto el sujeto $\mathrm{J} 12$ muestra un porcentaje de respuestas bimodal sobre 4 , el valor actual del programa, y sobre 6 , el valor de la condición previa; el sujeto $\mathrm{J} 9$ desarrolla una distribución de respuestas con un mayor porcentaje sobre el valor del programa.

En las Figuras 9 y 10, se presentan las gráficas que describen la dependencia secuencial de los ensayos para las condiciones de 6 a 8 y de 6 a 4 respuestas exactas respectivamente. En este análisis se intenta determinar probabilísticamente la influencia del resultado de un ensayo previo en el ensayo posterior. Debido a que las probabilidades de ocurrencia de las longitudes de carrera se agrupan muy cerca del valor del programa, se decidió realizar el análisis considerando únicamente los datos en los que las palomas hubiesen emitido una respuesta menos, un número igual o mayor en uno al valor del programa. Por ejemplo, en la gráfica superior izquierda de la Figura 9 , se muestra la ejecución del sujeto J 53 en la condición de 6 exacto. En este caso, después de emitir 5 respuestas en un ensayo, la probabilidad de 

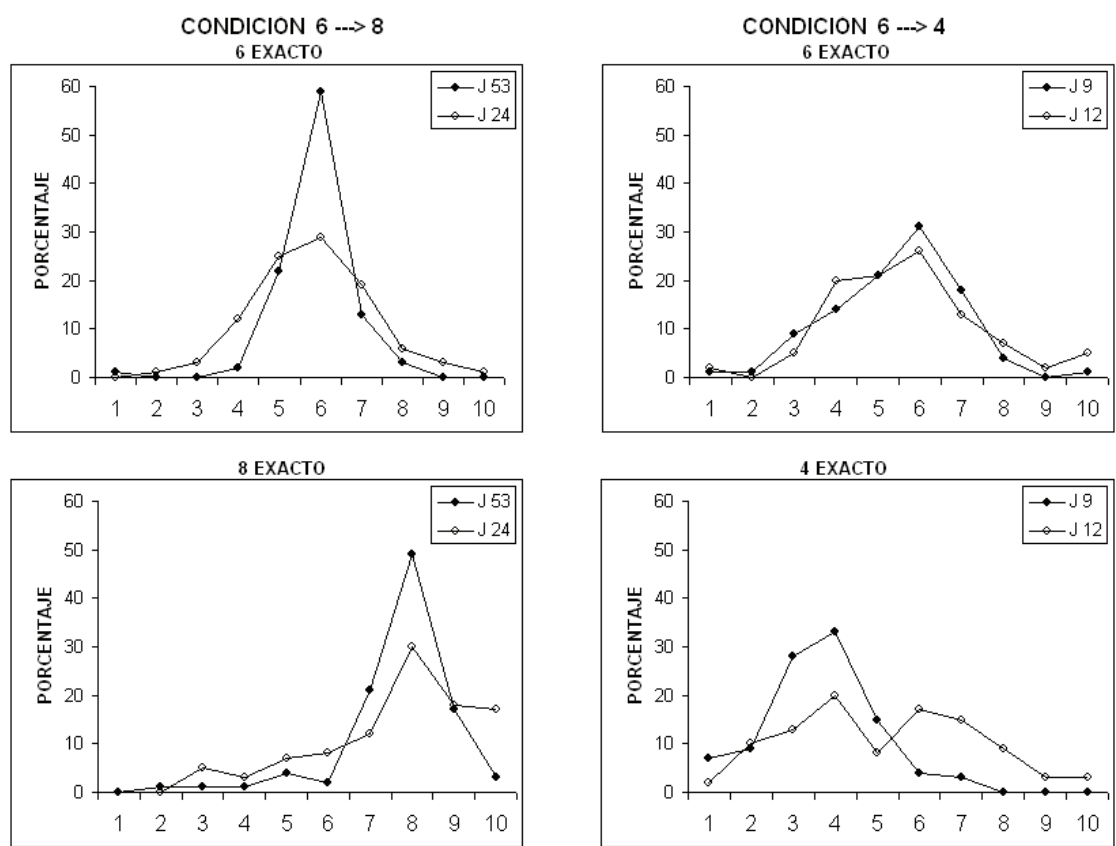

LONGITUD DE CARRERA

Figura 8. Muestra en la columna izquierda el porcentaje de respuesta para los sujetos en las condiciones experimentales de 6 y 8 exacto, y en la columna derecha el mismo dato para los sujetos con valores de 6 y 4 exacto; ambas gráficas como una función de la longitud de la carrera de respuestas en el Experimento 2.

presentar nuevamente 5 respuestas es $p=.32$, de emitir 6 respuestas en el siguiente ensayo es $p=.45$, y de presentar 7 respuestas es $p=.10$. La ejecución de los dos sujetos de la condición de 6 a 8 exacto muestra, con excepción del sujeto 24 en la condición de 8 exacto, un patrón semejante al descrito. En otras palabras, la ejecución muestra un incremento en la probabilidad de dar una respuesta más en el siguiente ensayo si el presente no ha sido reforzado, una probabilidad cercana a 0.5 de responder nuevamente con el mismo número de respuestas si el ensayo fue reforzado, y un decremento en el responder si el ensayo previo no fue reforzado por haber presentado una respuesta más que el número establecido por el programa. En la Figura 10 se presentan las gráficas de los animales que trabajaron en 6 a 4 exacto. La ejecución de la paloma J 9, en ambos valores del programa, fue semejante a 

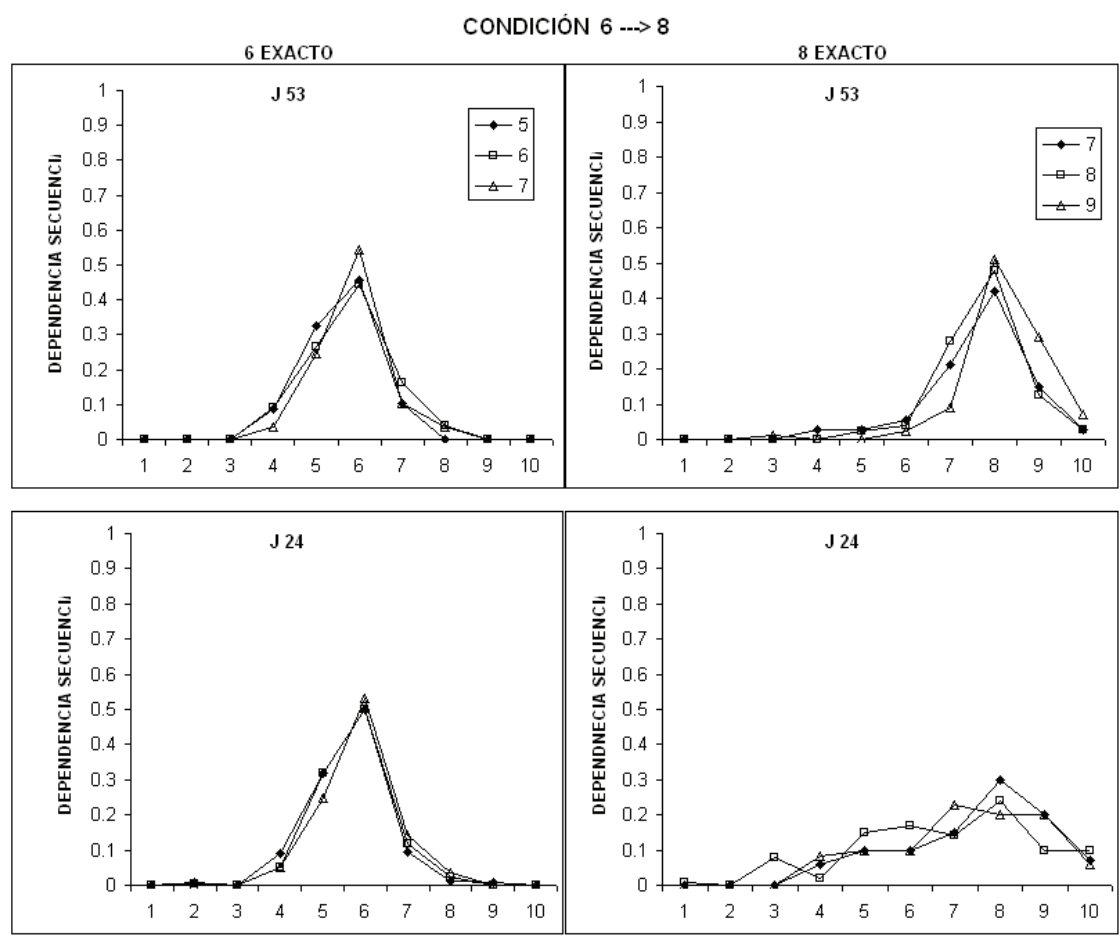

LONGITUD DE CARRERA

Figura 9. La dependencia secuencial en las condiciones experimentales de 6 y 8 exacto en el Experimento 2.

la mostrada por los sujetos de la condición de 6 a 8; a diferencia del sujeto $\mathrm{J}$ 12 , del cual se observa, en ambos valores del programa, cierta dificultad para utilizar el número de respuestas del ensayo previo como guía para emitir el número correcto en el ensayo posterior.

\section{DISCUSIÓN}

Son dos los efectos de mayor interés observados en este experimento. El primero se relaciona con la ejecución en las condiciones denominadas como rangos. Aquí se observa que los sujetos, incluso los que no participaron en las condiciones de discriminación absoluta de respuestas, muestran una tendencia a incrementar una respuesta en la longitud de carrera conforme el 

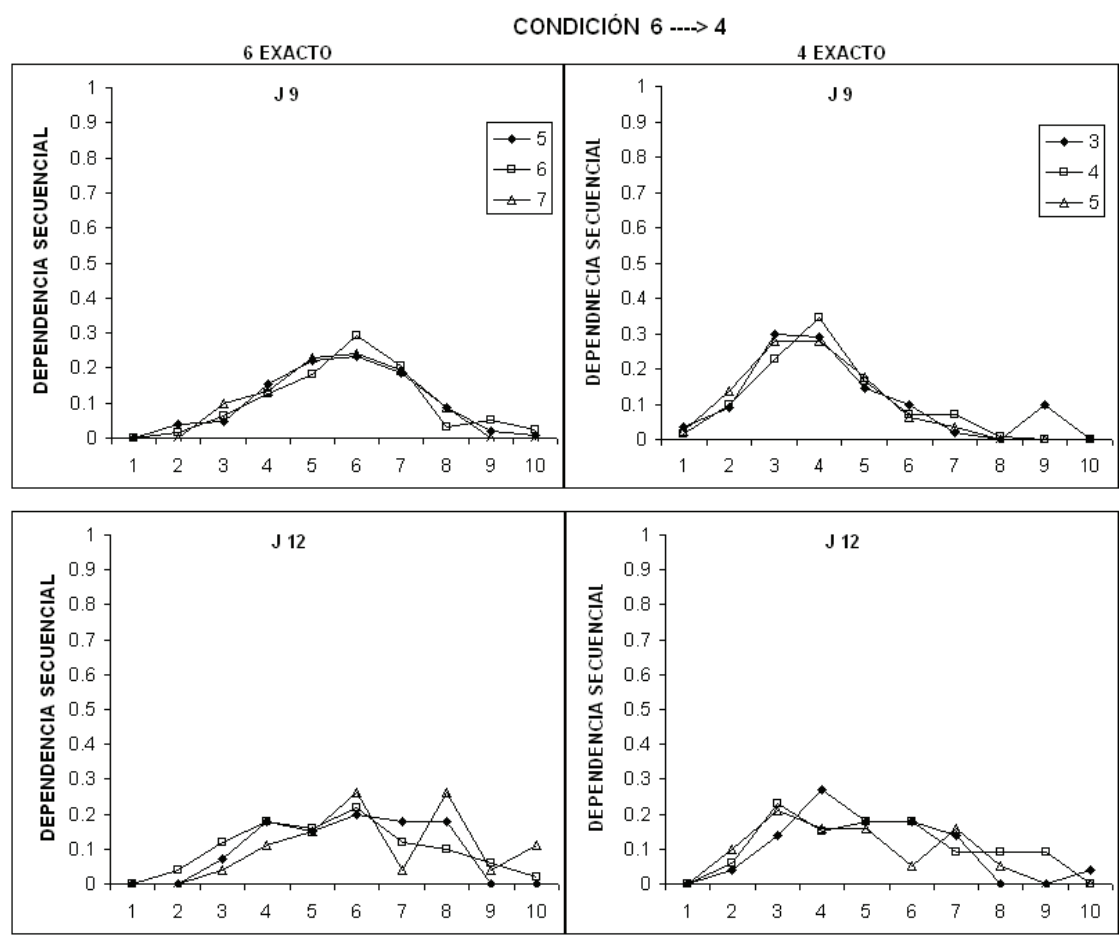

LONGITUD DE CARRERA

Figura 10. La dependencia secuencial en las condiciones experimentales de 6 y 4 exacto en el Experimento 2.

rango de respuesta a reforzar se hace más estrecho. Por ejemplo, cuando el rango se reduce de 3-9 a 4-8, las palomas que en general respondían más sobre el valor de 3 ahora lo hacen sobre 4 (Figura 7). La consistencia de este efecto a lo largo de las condiciones, muestra una discriminación entre valores adyacentes del programa, que aunque pudiera no ser tan precisa como una discriminación exacta o absoluta del número de respuestas, estaría cerca de lo que para algunos autores es considerado como conteo (Capaldi y Miller, 1988; Church y Meck, 1984; Thomas, Fowlkes y Vickery, 1980, ver a Davis y Pérusse, 1980 para un revisión). La razón para considerarlo así, es que en el conteo se requiere de discriminaciones categóricas entre números distintos, además de la asignación de una "etiqueta" conductual para cada valor discriminado. Este efecto es consistente también con los datos del Experimento 
1, en los cuales se encontró que la mayor probabilidad de ocurrencia de una longitud de carrera se ubica sobre o alrededor del valor mínimo de respuestas por reforzador, cuando esa fue la tarea para los sujetos.

El segundo resultado de interés es el efecto conocido como dependencia secuencial: la influencia del resultado en el ensayo previo sobre el ensayo posterior, en términos del número de respuestas emitidas. Este fenómeno, observado en la discriminación exacta del número de respuestas, tiene como único antecedente un estudio de Mechner (1958b) en el cual se empleó un sólo valor de un programa NFC. Los resultados del trabajo de Mechner, muestran que la longitud de una carrera se relaciona con la longitud de la carrera inmediata anterior. Este hallazgo está presente en el Experimento 2, aunque dadas las diferencias en la contingencia de reforzamiento entre un programa NFC, con un valor mínimo de respuesta y otro con el reforzador programado para un número exacto, los cambios observados en la ejecución en el Experimento 2 ocurren en dos direcciones: si el sujeto queda "corto" en el número de respuestas en el ensayo previo tiende a incrementar el número de éstas en el posterior, si queda "largo" entonces la tendencia es a emitir una cantidad menor de respuestas (Figuras 9 y 10 ).

Un efecto adicional, de la misma manera que en el Experimento 1, es que los sujetos mostraron dificultad para discriminar el valor del programa, cuando se les pidió en la segunda condición de exactos, emitir un número menor de respuestas en relación a un valor determinado previamente (Figura 10).

\section{DISCUSIÓN GENERAL}

Los resultados del presente estudio, muestran que la precisión en la discriminación numérica en palomas, está influida por el orden de presentación de los valores de programas basados en la respuesta y por el tipo de entrenamiento utilizado. Los resultados del Experimento 1, mostraron que las palomas que inician con un valor bajo para cambiar a un valor alto del programa NFC, ajustan más rápido y con mayor precisión el número mínimo de respuestas para obtener el reforzador. En el caso de los sujetos ubicados en la condición experimental inversa, cambiar de un valor alto a uno bajo representó una mayor dificultad para discriminar el segundo valor del programa. Este deterioro de la discriminación en la condición Descendente, afecta de manera proactiva al resto de las fases experimentales. En el Experimento 2, los cambios en los rangos de respuesta tuvieron como resultado, que la mayor probabilidad de ocurrencia de una determinada longitud de carrera se ubicara sobre el límite inferior del rango. Al analizar las dependencias secuenciales en la prueba de discriminación absoluta, se encontró que el resultado del ensayo previo sí tuvo un efecto en el ensayo posterior, que consistió en el incremento de 
la probabilidad de emitir un número mayor o menor de respuestas, dependiendo del número presentado en el ensayo anterior. El análisis de la discriminación del número absoluto de respuestas para obtener el reforzador, es un área prácticamente inédita en la investigación animal. Sin embargo, en el Experimento 2 se encontró evidencia de discriminación fina del número de respuestas, lo que confirma la hipótesis de que con un entrenamiento conveniente las palomas pueden realizar esa tarea.

Los resultados generales del presente estudio, permiten decir que las palomas discriminan numerosidades de respuestas, bajo condiciones experimentales en las que el único estímulo discriminativo disponible es su propia conducta y donde la decisión de finalizar los ensayos es independiente de la intervención del experimentador. En este sentido, las palomas demostraron la capacidad de estimar densidades de respuestas que les permiten detectar el número de éstas, ya sea mínimo o exacto, para obtener el reforzador. ¿Lo anterior significa que la discriminación observada en el Experimento 2 puede considerarse equivalente al conteo de los humanos?. La respuesta es, definitivamente no.

El conteo según Davis y Perusse (1988), es una habilidad numérica que requiere de la discriminación categórica entre distintos números, una etiqueta para cada valor discriminado. Con requisitos tan estrictos como éstos, es posible que el conteo esté restringido a pocas especies, en circunstancias particulares, y exceptuando a los humanos, a un número pequeño de objetos o eventos. Un aspecto interesante de los criterios mencionados por Davis y Pérusse (1988) sobre conteo, es que forman parte de un modelo desarrollado por Gelman y Gallistel (1978) producto de observaciones con infantes humanos. El modelo, provee de una serie de principios que sirven para calificar la forma en que los infantes humanos adquieren el concepto de número,definido como la capacidad de realizar combinaciones cuantitativas simples como sumar y restar.

En este sentido, quizá la única interrogante a la que se pudiera dar respuesta en el presente estudio, es la que cuestiona si los animales poseen el concepto de número, o para decirlo coloquialmente: ¿cuentan?. En este caso, nuevamente,la respuesta enfática es no; en general. Aunque siendo cautelosos, esta aseveración quizá dependa de la especie animal en estudio. Boysen y Bernston (1989), en el que posiblemente sea el mejor estudio sobre competencia numérica en animales, entrenaron a un chimpancé para que realizara operaciones aritméticas con números pequeños. El sujeto experimental mostró habilidad no sólo para realizar las operaciones requeridas, sino además transfirió la tarea aprendida con objetos a símbolos que representaban números arábigos; quizá sea éste el único caso conocido en el que un animal cumple con los principios postulados por Gelman y Gallistel (1978). Sin embargo, también es probable que para la mayoría de los animales, dis- 
criminaciones menos precisas de elementos sean suficientes para el análisis de las características cuantitativas del ambiente.

\section{REFERENCIAS}

Beninger, R.J., Kendall, S.B. y Vanderwolf, C.H.(1974). The ability of rats to discriminate their own behaviors. Canadian Journal of Psychology, 28 (1), 79-91.

Boysen S.T. y Bernston, G.G. (1989). Numerical competence in a chimpanzee (Pan troglodytes). Journal of Comparative Psychology, 103, 23-31.

Capaldi, E. J. y Miller, J. D. (1988). Counting in rats: its functional significance and the independent cognitive processes that constitute it. Journal of Experimental Psychology: Animal Behavior Processes, 4 (1), 3-17.

Church, R.M. y Meck, W.H. (1984) The numerical attribute of stimuli. In H.L. Roitblat, T.G. Bever, y H.S. Terrace (Eds.) Animal Cognition (pp. 445-464) Hillsdale, N.J: Lawrence Erlbaum Associates.

Davis, H. y Memmot J. (1982). Counting behavior in animals: a critical evaluation. Psychological Bulletin, 92, (3) 547-571.

Davis, H. y Pérusse, R. (1988). Numerical competence in animals: Definitional issues, current evidence, and a new research agenda. Behavioral and Brain Sciences, $11,561-615$.

Gallistell, C. R. y Gelman, R. (1992). Preverbal and verbal counting and computation. Cognition, 44, 43-74.

Gelman, R. y Gallistel, C.R. (1978). The child's understanding of number.Harvard University Press.

Hauser, M.D. (2000). Wild Minds: What animals really think. New York. Holt.

Hurwitz, H. M. B. (1962). Some properties of behavior under fixed-ratio and counting schedules. British Journal of Psychology, 53, 167-173.

Lionello-DeNolf, K.M. y Urcuioli, P.J. (2003). A procedure for generating differential "sample" responding without different exteroceptive stimuli. Journal Of Experimental Analysis of Behavior, 79, 21-35.

Krebs, J.R. (1978). Optimal foraging. Decision rules for predators. In J.R. Krebs y N.B. Davies (Eds.) Behavioural Ecology: An evolutionary approach. (pp. 23-63). Oxford, UK: Blackwell.

Matzusawa, T. (1985). Use of numbers by a chimpanzee. Nature, 267, 694-696.

Mechner, F. (1958a). Probability relations within response sequences under ratio reinforcement. Journal of the Experimental Analysis of Behavior, 1, 109-121.

Mechner, F.(1958b). Sequential dependencies of the lengths of consecutive response runs. Journal of the Experimental Analysis of Behavior, 1, 229-233.

Mechner, F.y Guevrekian, L.(1962). Effects of deprivation upon counting and timing in rats. Journal of the Experimental Analysis of Behavior, 5, 463-466.

Mechner, F. y Latranyi, M. (1963). Behavioral effects of caffeine, metamphetamine and methylphenidate in the rat. Journal of the Experimental Analysis of Behavior, 6, 331-342.

Meck, H. M. y Church, R. M. (1983). A mode control model of counting and timing processes. Journal of Experimental Psychology, 9, 320-334. 
Platt, J. R. y Johnson, D.M.(1971). Localization of position within a homogeneous behavior chain: effects of error contingencies. Learning and Motivation, 2, 386-414.

Shimp, C.P. (1982). On metaknowledge in the pigeon: An organism's knowledge about his own behavior. Animal Learning and Behavior, 10 (3), 358-364.

Shimp, C.P. (1983). The local organization of behavior: Dissociations between a pigeon's behavior and self reports of that behaviors. Journal of Experimental Analysis of Behavior, 39, 61-68.

Snodgrass, H.S., Hardin, L.J. y McMillan, E.D. (1997). Behavior rats under a fixed consecutive number schedules: effects of drugs of abuse. Journal of the Experimental Analysis of Behavior, 68, 117-132.

Thomas, R.K., Fowlkes, D. y Vickery, J.D. (1980). Conceptual numerousness judgments by squirrel monkeys. American Journal of Psychology, 93, 247-257. 